\title{
Fingolimod ameliorates cognitive impairments in a phencyclidine-induced rat model of schizophrenia
}

\author{
Tao $\mathrm{Li}^{1}$, Xueli $\mathrm{Yu}^{1}$, Xueyu Qi ${ }^{1}$, Long $\mathrm{Wei}^{2}$, Liansheng Zhao ${ }^{2}$, Wei Deng ${ }^{1}$, Wanjun Guo ${ }^{2}$, \\ Qiang $\mathrm{Wang}^{2}$, Xiaohong $\mathrm{Ma}^{2}$, Xun $\mathrm{Hu}^{1}$, and Peiyan $\mathrm{Ni}^{2}$ \\ ${ }^{1}$ Zhejiang University School of Medicine \\ ${ }^{2}$ Sichuan University West China Hospital
}

February 4, 2022

\begin{abstract}
Background and Purpose: Improvement of cognitive deficits in schizophrenia remains an unmet need due to the lack of new therapies and drugs. Recent studies have reported that fingolimod, an immunomodulatory drug for treating multiple sclerosis, demonstrates anti-inflammatory and neuroprotective effects in several neurological disease models. This suggests its usefulness for ameliorating cognitive dysfunction in schizophrenia. Herein, we assessed the efficacy profile and mechanism of fingolimod in a rat model of phencyclidine (PCP)-induced schizophrenia. Experimental Approach: Sprague-Dawley rats were treated with PCP for 14 days. The therapeutic effect of fingolimod on cognitive function was assessed using the Morris water maze and fear conditioning tests. Hippocampal neurogenesis and the expression of astrocytes and microglia were evaluated by immunostaining. Cytokine expression was quantified using multiplexed flow cytometry. Brain-derived neurotrophic factor expression and phosphorylation of extracellular signal-regulated kinase were determined using western blot analysis. Key Results: Fingolimod attenuated cognitive deficits and restored hippocampal neurogenesis in a dose-dependent manner in PCPtreated rats. Fingolimod treatment exerted anti-inflammatory effects by inhibiting microglial activation and IL- 6 and IL- $1 \beta$ pro-inflammatory cytokine expression. The underlying mechanism involves the upregulation of brain-derived neurotrophic factor protein expression and activation of the extracellular signal-regulated kinase signalling pathway. Conclusion and Implications: To the best of our knowledge, this is the first preclinical study to assess the effects of fingolimod on cognitive function in schizophrenia models. Our results support the role of the immune system in cognitive alterations in schizophrenia and highlight the potential of immunomodulatory strategies to improve cognitive deficits in schizophrenia.
\end{abstract}

\section{INTRODUCTION}

Schizophrenia is a severely debilitating mental disorder affecting approximately $1 \%$ of the global population. The disorder is characterized by various symptoms that can be categorised as positive symptoms (such as hallucinations and delusions), negative symptoms (such as avolition and self-neglect), and cognitive deficits (including deficits in memory and attention). Despite recent advances, the overall management of schizophrenia remains unsatisfactory. Cognitive deficits are among the symptoms of schizophrenia that are most difficult to treat. Therefore, the development of new therapies targeting cognitive dysfunction may represent the most demanding need among patients with schizophrenia.

The pathophysiology of schizophrenia is still not fully understood. However, emerging evidence suggests that the dysregulation of the immune system is linked to this disorder. Epidemiological studies have reported that patients with schizophrenia have an increased risk of autoimmune disorders (Cullen, Scarlett et al., 2018). Several genetic association studies have shown that, on average, patients with schizophrenia have an immune system that is more prone to activation (Sekar, Bialas et al., 2016; Woo, Pouget et al., 2020), which may have a detrimental effect on neurogenesis by reducing brain-derived neurotrophic factor (BDNF) 
expression (Lima Giacobbo, Doorduin et al., 2019). Furthermore, the loss of blood-brain barrier (BBB) integrity appears to be a common pathological finding in schizophrenia (Greene, Hanley et al., 2020), which leads to the infiltration of peripheral immune cells, culminating in neuroinflammation and oxidative stress. These disturbances are associated with disrupted glutamate homeostasis, impaired antipsychotic action, and exacerbated symptoms of schizophrenia (Pollak, Drndarski et al., 2018). These findings suggest that managing excessive pro-inflammatory processes may represent an important intervention target for this illness.

Fingolimod, a sphingosine-1-phosphate (S1P) receptor modulator, is highly efficacious against multiple sclerosis (MS). It acts on the S1P1 receptor of lymphocytes and selectively retains autoreactive lymphocytes in lymph nodes, thereby reducing damage to the central nervous system (CNS) (Hla \& Brinkmann, 2011). In addition to its prominent effects on lymphocyte recirculation, preclinical and clinical studies have investigated its therapeutic potential for other diseases. For example, a combination of fingolimod and alteplase attenuates reperfusion injury and improves clinical outcomes in patients with acute ischaemic stroke (Zhu, Fu et al., 2015). It has also been shown to improve depression-like behaviour in chronically stressed animal models (di Nuzzo, Orlando et al., 2015; Guo, Gan et al., 2020) and enhance learning and memory in Huntington's disease (Miguez, García-Díaz Barriga et al., 2015) and Alzheimer's disease models (Hemmati, Dargahi et al., 2013). As discussed above, the anti-inflammatory and neuroprotective properties of fingolimod suggest its beneficial effects in schizophrenia. Therefore, we aimed to investigate the efficacy profile and mechanism of fingolimod in a rat model of schizophrenia. To the best of our knowledge, this is the first preclinical study on the effects of fingolimod on the cognitive function in schizophrenia models.

\section{METHODS}

\subsection{Ethical statement}

All animal care and experimental procedures complied with the Animal Research: Reporting of In Vivo Experiments guidelines (Sert, Hurst et al., 2020) and the recommendations of the British Journal of Pharmacology (Lilley, Stanford et al., 2020). Male Sprague-Dawley rats (RRID: RGD_70508) weighing 200-230 $\mathrm{g}$ were used as subjects at the beginning of the test. Four were housed per cage $(470 \mathrm{~mm}$ length $\times 312 \mathrm{~mm}$ width $\times 260 \mathrm{~mm}$ height) under environmentally controlled conditions $\left(22^{\circ} \mathrm{C}, 12 \mathrm{~h}\right.$ dark $/ 12 \mathrm{~h}$ light cycle, and relative humidity of $60 \%$ ) with access to standard laboratory chow and water ad libitum. The animals were randomly allocated and acclimated for seven days before the experiments. All experimental procedures were performed in accordance with the Care and Use of Laboratory Animals guidelines (National Institutes of Health). The protocols were approved by the Animal Research Ethics Board of Zhejiang University.

\subsection{Group sizes}

The current experiment followed the rules for replacement, refinement, and reduction. Efforts were made to minimise suffering and reduce the number of animals used in the present study. The sample sizes were determined based on previous experience and reports of PCP-induced cognitive deficits in conditioned response tests (J. David Jentsch \& Taylor, 2001). In total, 64 rats were randomly divided into four groups. The drug treatment regimen and the experimental protocol are shown in Figure 1. We used 32 rats for the Morris water maze test ( $\mathrm{n}=8$ per group) and 32 rats for the fear conditioning test ( $\mathrm{n}=8$ per group). Two rats were excluded because they died on the first and fourth days of PCP treatment, respectively.

\subsection{Drug administration}

PCP, generously gifted by professors Haohong Li and Xiaohui Wang from the Changchun Institute of Applied Chemistry, Chinese Academy of Science. Fingolimod (Sigma-Aldrich, St. Louis, MO, USA, Cat\# SML0700), was dissolved in normal saline (NS) and freshly prepared daily. The dose regimen was based on an established protocol (Marquis, Audet, Doré, \& Goulet, 2007). The animals received a daily intraperitoneal (i.p.) injection of normal saline or PCP $(10 \mathrm{mg} / \mathrm{kg})$ for 14 days. After a seven-day wash-out period, the PCP-exposed rats received $0.5 \mathrm{mg} / \mathrm{kg}$ or $1 \mathrm{mg} / \mathrm{kg}$ fingolimod as the treatment group or NS as the PCP model group. Behavioural testing began at the end of the drug treatment (Figure 1). To label proliferating cells, the rats received an 
i.p. injection of $50 \mathrm{mg} / \mathrm{kg}$ BrdU (Sigma-Aldrich, St. Louis, MO, USA, Cat\# B5002) at the end of the Morris water maze test, and brain tissue was collected $24 \mathrm{~h}$ later.

\subsection{Morris Water Maze test}

Spatial learning and memory were evaluated using the Morris water maze, as described previously (Morris, 1984) with minor modifications. Briefly, the rats were trained for four consecutive days after the last injection of fingolimod or the vehicle. Each rat was trained to find a fixed platform (diameter: $10 \mathrm{~cm}$ ) hidden $1 \mathrm{~cm}$ underneath the water surface, and different geometric shapes on the surrounding walls served as visual cues. Each rat was randomly placed into the water at one of the three starting points and allowed $90 \mathrm{~s}$ to find the platform. The time to reach the platform (escape latency) was automatically recorded by the software. Rats that failed to reach the platform within $90 \mathrm{~s}$ were manually guided to the platform and allowed to stay on it for $10 \mathrm{~s}$. On the fifth day, the platform was removed and the rats were subjected to a probe trial session. Each rat was released from the opposite platform quadrant and allowed to swim for $60 \mathrm{~s}$. The swimming path, virtual platform crossing time, time spent in each quadrant, and swimming speed of each rat were recorded. Video signals were analysed using EthoVision 7.0 (Noldus Information Technology, Leesburg, VA, RRID: SCR_000441).

\subsection{Fear conditioning test}

Fear conditioning procedures were performed according to previous protocols, with several modifications (Nassireslami, Nikbin et al., 2013). The tests took place in a $26.5 \mathrm{~cm} \times 27.5 \mathrm{~cm} \times 41.5 \mathrm{~cm}$ chamber (UGO BASILE SRL, Gemonio, VA, Italy) with clear Plexiglas walls and a ceiling with holes to allow ventilation. The floor was wired to a shock source and solid-state grid scrambler for the delivery of the foot shock. The chamber was illuminated by a single house light positioned at the top centre of one wall. A video camera was suspended on top of the chamber to record the behaviour of the animals, and an audio generator was mounted at the corner of the back wall. Before conditioning was initiated, the rats were brought to the behavioural assessment room for $1 \mathrm{~h}$, placed individually in the conditioning box for $10 \mathrm{~min}$, and returned to their home cages. This habituation procedure was introduced thoroughly to familiarise the rats with the stimuli of the experimental room, thus preventing any interference from uncontrolled novel stimuli during the experiments. The rats were trained and tested for three days, placed in the chamber on the day of conditioning, and allowed to explore freely for $2 \mathrm{~min}$. After the habituation procedure, an electric foot shock $(0.5 \mathrm{~mA}, 2 \mathrm{~s})$ was delivered immediately after a tone $(3600 \mathrm{kHz}, 20 \mathrm{~s})$. The second cue tone and foot shock were delivered $2 \mathrm{~min}$ later. After termination of the shock, the rats were allowed to stay in the chamber for another 2 min before returning to their home cage. The contextual cued fear test was performed on day 2 . The rats were, subsequently, returned to the conditioning box. Their behaviours were recorded for 3 min without a tone or foot shock, and the freezing time was automatically recorded. Freezing was defined as the complete absence of movement except for respiratory motion. The auditory-cued fear test was performed on day 3 in an altered chamber. Each rat was placed in a modified chamber and allowed to explore for 3 min, the cue tone $(3600 \mathrm{kHz}, 20 \mathrm{~s})$ was delivered, and the freezing time was recorded for an additional 3 min. Freezing was presented as a percentage of the observation period. Data were recorded and analysed using a video tracking software (ANY-MAZE, Stoelting Co., Wood Dale, IL, USA; RRID: SCR_014289).

\subsection{Western blot}

The immunoblotting-based procedures in this study complied with the recommendations of the British Journal of Pharmacology (Alexander, Roberts et al., 2018). The rats were sacrificed by decapitation after isoflurane anaesthesia, and bilateral hippocampal tissue from each group was quickly extracted in ice-cold radio-immunoprecipitation assay lysis buffer (Life Technologies Co. USA; Cat\# 89901). Proteins extracted from the hippocampal tissue were loaded on a 10\% SDS-PAGE gel and blotted onto a hydrophobic polyvinylidene fluoride transfer membrane (0.45 $\mu \mathrm{m}$ pore size, Millipore, Billerica, MA, USA, Cat\# IPVH00010). After being blocked with $5 \%(\mathrm{w} / \mathrm{v})$ non-fat milk in Tris-buffered saline with $0.1 \%$ Tween-20 for $1 \mathrm{~h}$, the membranes were probed with primary antibodies in blocking buffer at $4^{\circ} \mathrm{C}$ overnight and incubated with the horseradish peroxidase-conjugated secondary antibodies (Cell Signaling Technology, USA; 1:2000 Cat\# 7074, polyclo- 
nal anti-rabbit IgG, RRID: AB_2099233, Cat\# 7076, polyclonal anti-mouse IgG, RRID: AB_330924) for 1 $\mathrm{h}$ at room temperature. Immunoreactive antigens were detected using chemiluminescence agents (Bio-Rad Laboratories, Western ECL Blotting Substrates, RRID: SCR_008426) and visualised using the ChemiDoc XRS System with Image Lab Software (RRID: SCR_014210, Bio-Rad Laboratories, USA). Optical densities were analysed using ImageJ software (RRID: SCR_003070, NIH, Bethesda, Maryland, USA). The primary antibodies were diluted in $5 \%$ bovine serum albumin blocking buffer as follows: rabbit polyclonal IgG antip-ERK1/2 (Cell Signaling Technology, USA; 1:1000, Cat\# 9101, RRID: AB_331646), rabbit polyclonal IgG anti-total ERK1/2 (Cell Signaling Technology, USA; 1:1000, Cat\# 9102, RRID: AB_330744), rabbit monoclonal IgG anti-BDNF (Abcam, Cambridge, USA; 1:1000, Cat\# ab108319, RRID: AB_10862052). Mouse monoclonal IgG anti- $\beta$-actin (Proteintech, USA; 1:8000, Cat\# CL488-66009, RRID: AB_2883225) was used as the loading control, and the data were expressed as relative normalised expression and analysed by an independent researcher.

\subsection{Tissue preparation and immunofluorescence staining}

The immunohistochemistry procedures followed the recommendations by the British Journal of Pharmacology (Alexander, Roberts, Broughton, Sobey, George, Stanford et al., 2018). Rats from each group (n=7) were deeply anaesthetised and transcardially perfused with $0.9 \%$ saline, followed by $4 \%$ paraformaldehyde solution. The brains were fixed in $4 \%$ paraformaldehyde, transferred to $30 \%$ sucrose solution for 5 days, and embedded in OCT at -20 . Serial coronal sections $(30 \mu \mathrm{m})$ were obtained using a cryostat microtome (Leica, Wetzlar, Germany). Brain slices were washed three times with phosphate-buffered saline (PBS), permeabilised with $0.1 \%$ Triton X-100 for 10 min, blocked in $10 \%$ goat serum (Invitrogen Corp. USA, Cat\# 16210064 ) for $1 \mathrm{~h}$ at room temperature, and incubated with the primary antibody overnight at $4^{\circ} \mathrm{C}$. For the detection of BrdU-labelled nuclei, the specimens were previously incubated in $2 \mathrm{~N} \mathrm{HCl}$ at 37 , followed by a 10-min rinse in $0.1 \mathrm{M}$ sodium tetraborate $\mathrm{pH} 8.5$ and two rinses with PBS before the blocking step. The following primary antibodies were diluted in blocking solutions: mouse monoclonal IgG anti-BrdU (Biolegend, USA; 1:250, Cat\# 339802, RRID: AB_1595495), rabbit monoclonal IgG anti-NeuN (Cell Signaling Technology, USA; 1:500, Cat\# 24307, RRID: AB_2651140), rabbit monoclonal IgG anti-doublecortin (DCX, Cell Signaling Technology, USA; 1:500, Cat\# 4604, RRID: AB_561007), mouse monoclonal IgG anti-glial fibrillary acidic protein (GFAP, Sigma, USA; 1:500, Cat\# G3893, RRID: AB_477010), and rabbit polyclonal IgG anti-Iba1 (Proteintech, China; 1:500, Cat\# 10904-1-AP, RRID: AB_2224377). The brain slices were washed with PBS before incubation with Alexa Flour-conjugated secondary antibodies (Alexa Fluor 555-conjugated donkey anti-mouse IgG, Cat\# A-31572, RRID: AB_162543; Alexa Fluor 488-conjugated donkey anti-rabbit IgG, Invitrogen Corp. USA; 1:500, Cat\# A-21202, RRID: AB_141607) and DAPI (4', 6-diamidino-2-phenylindole, Invitrogen Corp. USA; 1:1000; RRID: AB_2307445) for $1 \mathrm{~h}$ at room temperature. Finally, the sample slices were mounted on glass slides using a fluorescence mounting medium (DAKO, Danmark, Cat\# S3023, RRID: SCR_013530).

\subsection{Multiplex analysis of cytokines}

Quantification of IL-6 and IL-1 $\beta$ cytokines in hippocampal samples was performed as described previously (Sanchez-Alavez, Nguyen et al., 2019) by a multiplexed flow cytometric assay using a rat cytokine kit (Millipore, Billerica, MA, USA, Cat\# RECYTMAG-65K) according to the manufacturer's instructions. Briefly, hippocampal tissue lysate and pre-mixed antibody beads were incubated at room temperature for $2 \mathrm{~h}$, washed three times, and incubated with streptavidin-phycoerythrin for $30 \mathrm{~min}$. The contents were washed and resuspended in sheath fluid before being read on the Luminex MAGPIX system. The results were analysed using MILLIPLEX Analyst 5.1 software (Luminex Corporation, Austin, TX, USA). The concentrations of cytokines were determined based on standard curves, and the coefficient of variation was calculated; it did not exceed $20 \%$.

\subsection{Cell counts and quantification}

A modified unbiased stereology protocol was used for BrdU, DCX, Iba-1, and GFAP quantification (Malberg, Eisch et al., 2000). One out of every six adjacent sections throughout the hippocampus was chosen and 
processed for immunohistochemistry. The number of immunopositive cells in the dentate gyrus layer and hilus throughout the entire hippocampus was imaged using a Zeiss Axio Imager A2 microscope (Carl Zeiss, Jena, Germany, RRID: SCR_018876) or Pannoramic 250 Flash digital scanners (3D HISTECH Ltd, Budapest, Hungary). The results were expressed as the average number of positive cells per section and analysed using Image-Pro Plus 6.0 software (Media Cybernetics, Silver Spring, USA, RRID: SCR_007369) by an independent researcher.

\subsection{Data analyses}

The data and statistical analyses complied with the experimental design and analysis recommendations in pharmacology (Curtis, Alexander et al., 2018). The sample size for statistical analysis was at least five animals per group. The data were analysed by researchers who were blinded to the origin of the data. The declared group size was the number of independent samples, and statistical analysis was performed using these independent values. No data were excluded from the statistical analysis. For western blot analysis, each sample was run in triplicate; the average value of the control group was depicted as "1", the other groups were normalised to the fold of the control mean. The Shapiro-Wilk normality test was used to assess the normality of the data. The effect of fingolimod treatment on escape latency in the Morris water maze was analysed using two-way repeated-measures analysis of variance (ANOVA). For non-parametric data, the Kruskal-Wallis test was used, followed by Dunn's post hoc test. All other comparisons were performed using one-way ANOVA followed by Dunnett's post hoc test. Post hoc tests were conducted only if the KruskalWallis test or F in ANOVA achieved the necessary level of statistical significance and no significant variance in homogeneity. The data are expressed as the mean \pm standard error of the mean and were analysed using Prism 8 (GraphPad Software Inc., San Diego, CA, USA; RRID: SCR_002798). Differences were considered statistically significant at $\mathrm{p}<.05$.

\section{RESULTS}

\subsection{Fingolimod attenuated PCP-induced spatial memory deficit in rats in the Morris water} maze

The results of the Morris water maze test showed significant main effects of treatment and number of testing days. Overall, latencies to reach the platform decreased for all groups over the 4 training days. Compared with the control, PCP-treated $(10 \mathrm{mg} / \mathrm{kg}$, i.p.) rats took a significantly longer time to reach the platform, and fingolimod-treated $(0.5 \mathrm{mg} / \mathrm{kg}, 1 \mathrm{mg} / \mathrm{kg}$, i.p. $)$ rats spent significantly less time to find the platform from day 2 (Figure $2 \mathrm{~A}$ ). In the probe test, the crossing times of the virtual platform and the time spent in the target quadrant for the PCP-treated rats were significantly lower than those for the control, indicating impairments in spatial learning and memory function. This deficit was attenuated by fingolimod treatment $(0.5 \mathrm{mg} / \mathrm{kg}, 1 \mathrm{mg} / \mathrm{kg}$, i.p.) in a dose-dependent manner (Figure 2B, C). Swimming speed showed no significant difference in the groups (Figure 2D). These results indicated that fingolimod effectively reversed spatial memory impairment in PCP-treated rats.

\subsection{Fingolimod attenuated PCP-induced contextual and tone memory retention deficits in rats in the fear conditioning task}

Next, we used a contextual fear conditioning task to evaluate the effects of fingolimod on memory retention. A timeline of the experiment is presented in Figure 3A. The rats were subjected to consecutive tone-shock pairings in a conditioning training session and tested for contextual and tone-cued fear conditioning tests 24 and $48 \mathrm{~h}$ later, respectively. We found that the percentage of freezing time for the PCP-treated $(10 \mathrm{mg} / \mathrm{kg}$, i.p.) rats was significantly lower than that for the control rats, indicating impairments in fear memory retention. Fingolimod treatment $(0.5 \mathrm{mg} / \mathrm{kg}, 1 \mathrm{mg} / \mathrm{kg}$, i.p.) significantly increased the freezing time in a dose-dependent manner (Figure 3B). Similar effects were observed in the auditory-cued fear conditioning test, although they were not statistically significant. The decreased freezing time in PCP-treated rats was partially restored by fingolimod treatment $(0.5 \mathrm{mg} / \mathrm{kg}, 1 \mathrm{mg} / \mathrm{kg}$, i.p.) (Figure 3C). These results suggest that fingolimod attenuates PCP-induced deficits in fear memory retention. 


\subsection{Fingolimod treatment enhanced neurogenesis in the dentate gyrus of PCP-treated rats}

Since the learning and memory processes are closely related to hippocampal neurogenesis (Deng, Aimone et al., 2010), and fingolimod ameliorates hippocampal-dependent behavioural tasks, we reasoned that the potential effects of fingolimod may be involved in neurogenesis. Therefore, we performed BrdU, NeuN, and DCX staining to assess neurogenic potential in the hippocampus (Figure 4A). The results showed that the number of BrdU- (Figure 4B), BrdU/NeuN- (double-positive) (Figure 4C), and DCX-positive (Figure $4 \mathrm{D})$ in the PCP-treated $(10 \mathrm{mg} / \mathrm{kg}$, i.p.) group was significantly lower than that in the control, indicating impairments of hippocampal neurogenesis. As expected, fingolimod treatment $(0.5 \mathrm{mg} / \mathrm{kg}, 1 \mathrm{mg} / \mathrm{kg}$, i.p.) significantly reversed this neurogenesis deficit by promoting neuronal proliferation in a dose-dependent manner.

\subsection{Fingolimod treatment inhibited microglial activation in the dentate gyrus of PCP-treated rats}

Accumulating evidence suggests that alterations in glial cells are closely related to the pathophysiology of schizophrenia (Agdm, Psaga et al., 2020). We hypothesised that the treatment effect of fingolimod may also be related to its immunomodulatory function. To test this hypothesis, we performed immunostaining of GFAP-labelled astrocytes and Iba-1-labelled microglia to evaluate glial activation in the hippocampus (Figure 5A). The results indicated that PCP $(10 \mathrm{mg} / \mathrm{kg}$, i.p.)-treated rats showed a significantly higher number of Iba-1-labelled microglia (Figure $5 \mathrm{~B})$, whereas fingolimod treatment $(0.5 \mathrm{mg} / \mathrm{kg}, 1 \mathrm{mg} / \mathrm{kg}$, i.p.) dose-dependently decreased the Iba-1 positive cells. However, no significant difference in GFAP expression was observed between the groups (Figure $5 \mathrm{C}$ ). The above results indicate that fingolimod treatment inhibited microglial activation in the dentate gyrus of PCP-treated rats.

$3.5 \Phi$

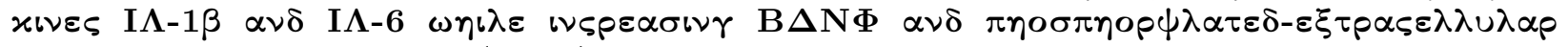

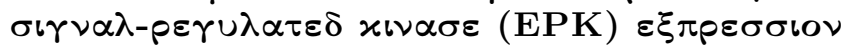

Since glial activation can produce pro-inflammatory cytokines, such as IL-6 and IL-1 $\beta$, which are thought to be trait markers of schizophrenia (Momtazmanesh, Zare-Shahabadi et al., 2019), we further examined cytokine expression in the hippocampus. We found that PCP-treated rats showed significantly higher IL-6 and L-1 $\beta$ levels. Concurrently, fingolimod $(0.5 \mathrm{mg} / \mathrm{kg}, 1 \mathrm{mg} / \mathrm{kg}$, i.p. $)$ partially attenuated the expressions of these pro-inflammatory cytokines in a dose-dependent manner (Figure $6 \mathrm{~A}, \mathrm{~B}$ ). The above results suggest that the BDNF/ERK pathway activation and inhibition of IL-1 $\beta$ and IL-6 cytokine expressions is involved in the treatment effect of fingolimod. The BDNF/ERK pathway is extensively involved in learning and memory processes and plays a crucial role in modulating synaptic plasticity and adult neurogenesis (Kowiański, Lietzau et al., 2018). To evaluate whether the treatment effect of fingolimod was due to activation of the BDNF/ERK pathway, we assessed the expressions of BDNF and phosphorylated ERK. The results indicated that BDNF protein expression and ERK1/2 phosphorylation in the PCP (10 mg/kg, i.p.)-treated group were decreased. In contrast, fingolimod treatment $(0.5 \mathrm{mg} / \mathrm{kg}, 1 \mathrm{mg} / \mathrm{kg}$, i.p.) increased BDNF expression (Figure $6 \mathrm{C}$ ) and ERK1/2 phosphorylation (Figure 6D) in a dose-dependent manner.

\section{DISCUSSION}

In the present study, we found that fingolimod, an S1P receptor modulator, attenuated PCP-induced impairments of spatial and fear memory by promoting neurogenesis and attenuating glial activation and IL-6 and IL-1 $\beta$ pro-inflammatory cytokine expressions. The underlying mechanism involves the upregulation of BDNF expression and activation of the ERK signalling pathway. The above results demonstrate that fingolimod is a potential therapeutic drug for treating cognitive impairment in schizophrenia.

Increasing evidence supports the idea that the dysregulated glutamatergic system plays a crucial role in the development of schizophrenia (Dauvermann, Lee et al., 2017), and administration of the N-methyl-D-aspartic acid receptor antagonist PCP, especially chronic use, induces typical symptoms in humans similar to those observed in patients with schizophrenia (J. D. Jentsch \& Roth, 1999). This has been the basis for inducing 
animal models that mimic the symptoms of schizophrenia. Consistent with previous studies, our study showed that chronic PCP (10 mg/kg, 14 days) treatment induced spatial and fear memory impairments that correlated well with those thought to occur in schizophrenia (Cadinu, Grayson et al., 2017).

Since learning and memory processes are closely related to hippocampal neurogenesis (Deng, Aimone, \& Gage, 2010) and fingolimod has been shown to facilitate hippocampal neurogenesis through the internalisation of the S1P1 receptor in hippocampal neural stem cells (NSC)s of adult mice (Yili Sun, Hong et al., 2016) (Efstathopoulos, Kourgiantaki et al., 2015), we reasoned that the treatment effect of fingolimod may be, at least in part, due to modulation of neurogenesis. In line with our animal behavioural tests, PCP treatment suppressed the proliferation and differentiation of NSCs, as indicated by the decreased number of BrdU-, NeuN-, and DCX-positive cells in the dentate gyrus. In contrast, fingolimod ameliorated this neurogenic deficit by facilitating adult neurogenesis.

Recently, the potential involvement of the immune response and consequent neuroinflammation in the pathogenesis of schizophrenia has attracted more attention. Similar to MS, schizophrenia is often considered to be associated with the dysregulation of the immune response and chronic inflammation in the CNS (Pape, Tamouza et al., 2019). In contrast, schizophrenia-like symptoms have also been described in some patients with MS (Meier, Ramagopalan et al., 2020). This suggests that the dysregulated immune system and inflammation observed in schizophrenia and MS somewhat overlap. Therefore, the aetiologies and therapeutic implications may be shared between both diseases.

Resident myeloid cells of the brain, microglia, astrocytes, and inflammatory cytokines are involved in mediating neuroinflammation. Similar to macrophages, microglia account for approximately $10 \%$ of all cells in the CNS (Soulet \& Rivest, 2008). They are thought to originate from myeloid precursor cells during early neonatal development and are the principal resident innate immune cells of the CNS (Santambrogio, Belyanskaya et al., 2001). Under normal circumstances, microglia maintained a downregulated phenotype. When exposed to stimuli such as chronic stress, injury, or inflammation, the microglia can be "activated" or "sensitized," by which a lower level of the same stimulus, such as cytokine response, can cause them to proliferate and increase the production of pro-inflammatory cytokines (Norbert \& Müller, 2018; Rahimian, Wakid et al., 2021). This sensitisation process heightens responsiveness to inflammatory signals, inducing psychopathological symptoms and cognitive deficits by exacerbating or re-exacerbating the inflammatory pathology in the CNS. Postmortem studies have found a significant increase in microglial density in the brains of patients with schizophrenia despite substantial heterogeneity between studies (Kesteren, Gremmels et al., 2017). In line with these findings, our study found significantly more Iba-1-labelled microglia in the hippocampus of PCP-treated rats, which may indicate a vulnerable environment for an inflammatory insult. Astrocytes are known to provide neurotrophic support for neurones, maintain a homeostatic environment, and modulate synapse pruning (Clarke, Laura et al., 2013). However, little is known about their functional changes in response to immune attacks or neuroinflammation (referred to as "reactive astrocytes"). Studies have shown that microglia can cause astrocytes to lose their normal functions and become neurotoxic, contributing to neuronal death (Liddelow, Guttenplan et al., 2017). In our study, a higher expression of GFAP-positive astrocytes was also found in the hippocampus, which suggested a highly hostile environment for neural function. Fingolimod may have an ameliorative effect by suppressing microglial and astroglial activation.

The presence of the BBB creates an "immune privileged" environment for the brain, shielding the CNS from neurotoxic insults from peripheral immune cells, cytokines, and chemokines. However, the loss of BBB integrity appears to be a common pathological finding in patients with schizophrenia (Greene, Hanley, \& Campbell, 2020), which often leads to an increase in the infiltration of the brain parenchyma and proinflammatory cytokine release. This possibly links increased neuroinflammation to detrimental effects on memory, neural plasticity, and neurogenesis. Among these, IL-6 and IL-1 $\beta$ appear to play essential roles in the pathogenesis of schizophrenia, and their findings are consistent. Both drug-naïve (Upthegrove, ManzanaresTeson et al., 2014) and chronic schizophrenia patients (Goldsmith, Rapaport et al., 2016) demonstrate higher expressions of IL- 6 and IL- $1 \beta$ than healthy controls. It has been demonstrated that the overproduction of IL- 6 
and IL-1 $\beta$ may result in aberrant hippocampal neurogenesis and cognitive impairment (Vallières, Campbell et al., 2002) (M. Wu, 2013). In line with these findings, we found higher levels of IL-6 and IL-1 $\beta$ in the hippocampus of PCP-treated rats, which were negatively correlated with their neurogenic potential. In contrast, fingolimod partially attenuated the IL-1 $\beta$ and IL-6 levels in a dose-dependent manner.

BDNF is one of the neurotrophins that plays a significant role in neurogenesis, synaptic plasticity, and cognitive function (Kowiański, Lietzau, Czuba, Waśkow, Steliga, \& Moryś, 2018). It is also involved in an upstream ERK cascade. The essential role of ERK activation in the CNS has been well-described in synaptic plasticity and memory (Iii, 2008). Multiple lines of evidence indicate that peripheral levels of BDNF are reduced in both drug-naïve and medicated patients with schizophrenia compared with healthy controls. (Green, Matheson et al., 2011). Although the interactions between BDNF and inflammatory cytokines require further elucidation, it has been demonstrated that patients with higher levels of inflammatory cytokines have lower BDNF expression in both postmortem and blood sample studies (Fillman, Cloonan et al., 2013; Valeria, Mondelli et al., 2011). A recent study found that aging-related and lipopolysaccharide-induced microglial activation in mice could be reversed by BDNF supplementation (S.-Y. Wu, Pan et al., 2020). In our study, ERK signalling was downregulated in parallel with BDNF expression in PCP-treated rats, which also suggests that PCP can induce cognitive deficits by compromising neurogenesis via the reduction of BDNF signalling. Furthermore, since a previous study showed that fingolimod-induced neurogenesis was dependent on the activation of ERK signalling (Y. Sun, Feng et al., 2016), we reasoned that the overall improvement in learning and memory function may be related to the restoration of BDNF/ERK signalling in PCP-treated rats.

It should be noted that an eight-week, double-blind pilot study was recently conducted to assess the effects of fingolimod in patients with schizophrenia and schizoaffective disorder (Francis, Hummer et al., 2020), which proposed that dysregulated inflammatory processes may contribute to the white matter abnormalities observed in schizophrenia. By analysing the fractional anisotropy values in diffusion tensor imaging, a significant relationship was found between the degree of lymphocyte reduction and improved white matter microstructure in the corpus callosum and the right superior longitudinal fasciculus. Significant improvements in the Brief Assessment of Cognition in Schizophrenia were not demonstrated; this may be partly due to the small sample size (40 patients in total) and the complex medication history of the recruited patients. However, studies involving larger samples and longer treatment durations are required to clarify the potential therapeutic effects of fingolimod in schizophrenia.

Some limitations of this study should be noted. The complex and unclear pathogenesis of schizophrenia is considered to be an outcome of gene-gene and gene-environment interactions; therefore, the current animal model based on N-methyl-D-aspartic acid receptor manipulation is focused on the cognitive deficits of schizophrenia, and cannot completely mirror the complex and heterogeneous aetiology of this disease. Therefore, other animal models of schizophrenia which adequately replicate "positive-like" symptoms (e.g., hyperactive stimulant sensitivity) and "negative-like" symptoms (e.g., social withdrawal) are needed to fully assess the treatment effects of fingolimod. In addition, for planning personalised treatment, further study is needed to help discover potential biomarkers of therapeutic relevance, so that we can identify the types of patients for whom fingolimod therapy may be suitable.

In conclusion, our study demonstrated that fingolimod improved PCP-induced learning and memory deficits in a rat model of schizophrenia. We highlight the ability of fingolimod to modulate neurogenesis and neuroinflammatory processes, which are markedly altered in schizophrenia. The study also showed that the BDNF/ERK pathway influenced the treatment effect of fingolimod. To the best of our knowledge, this is the first preclinical evidence supporting fingolimod as a potential therapeutic agent for cognitive deficits in schizophrenia.

\section{REFERENCES}

Agdm, A., Psaga, B., \& Pmna, B. (2020). Glial cells in schizophrenia: a unified hypothesis.

Alexander, S. P. H., Roberts, R. E., Broughton, B. R. S., Sobey, C. G., George, C. H., Stanford, S. C., . . 
. Ahluwalia, A. (2018). Goals and practicalities of immunoblotting and immunohistochemistry: A guide for submission to the British Journal of Pharmacology. British Journal of Pharmacology, 175 (3), 407-411. doi: https://doi.org/10.1111/bph.14112

Cadinu, D., Grayson, B., Podda, G., Harte, M. K., \& Neill, J. C. (2017). NMDA receptor antagonist rodent models for cognition in schizophrenia and identification of novel drug treatments, an update. Neuropharmacology, 142.

Clarke, Laura, E., Barres, Ben, \& A. (2013). Emerging roles of astrocytes in neural circuit development. Nature Reviews Neuroscience, 14 (6), 442-442.

Cullen, A. E., Scarlett, H., Pollak, T. A., Graham, B., Joyce, D. W., Kempton, M. J., . . Valeria, M. (2018). Associations Between Non-Neurological Autoimmune Disorders and Psychosis: A Meta-Analysis. Biological Psychiatry, 85, S0006322318316305-.

Curtis, M. J., Alexander, S., Cirino, G., Docherty, J. R., George, C. H., Giembycz, M. A., . . . Ahluwalia, A. (2018). Experimental design and analysis and their reporting II: updated and simplified guidance for authors and peer reviewers. British Journal of Pharmacology, 175 (7), 987-993. doi: https://doi.org/10.1111/bph.14153

Dauvermann, M. R., Lee, G., \& Dawson, N. (2017). Glutamatergic regulation of cognition and functional brain connectivity: insights from pharmacological, genetic and translational schizophrenia research. British Journal of Pharmacology, 174 (19), 3136-3160. doi: https://doi.org/10.1111/bph.13919

Deng, W., Aimone, J. B., \& Gage, F. H. (2010). New neurons and new memories: how does adult hippocampal neurogenesis affect learning and memory? Nature Reviews Neuroscience, 11 (5), 339-350. doi: $10.1038 / \mathrm{nrn} 2822$

di Nuzzo, L., Orlando, R., Tognoli, C., Di Pietro, P., Bertini, G., Miele, J., . . Nicoletti, F. (2015). Antidepressant activity of fingolimod in mice. Pharmacology Research 83 Perspectives, 3 (3), e00135. doi: https://doi.org/10.1002/prp2.135

Efstathopoulos, P., Kourgiantaki, A., Karali, K., Sidiropoulou, K., Margioris, A. N., Gravanis, A., \& Charalampopoulos, I. (2015). Fingolimod induces neurogenesis in adult mouse hippocampus and improves contextual fear memory. Translational Psychiatry, 5 (11), e685-e685. doi: 10.1038/tp.2015.179

Fillman, S. G., Cloonan, N., Catts, V. S., Miller, L. C., Wong, J., McCrossin, T., . . Weickert, C. S. (2013). Increased inflammatory markers identified in the dorsolateral prefrontal cortex of individuals with schizophrenia. Molecular Psychiatry, 18 (2), 206-214. doi: 10.1038/mp.2012.110

Francis, M. M., Hummer, T. A., Liffick, E., Vohs, J. L., \& Breier, A. (2020). Effects of fingolimod, a sphingosine-1-phosphate (S1P) receptor agonist, on white matter microstructure, cognition and symptoms in schizophrenia. Brain Imaging and Behavior .

Goldsmith, D. R., Rapaport, M. H., \& Miller, B. J. (2016). A meta-analysis of blood cytokine network alterations in psychiatric patients: comparisons between schizophrenia, bipolar disorder and depression. Molecular Psychiatry .

Green, M. J., Matheson, S. L., Shepherd, A., Weickert, C. S., \& Carr, V. J. (2011). Brain-derived neurotrophic factor levels in schizophrenia: a systematic review with meta-analysis. Mol Psychiatry, 16 (9), 960-972.

Greene, C., Hanley, N., \& Campbell, M. (2020). Blood-brain barrier associated tight junction disruption is a hallmark feature of major psychiatric disorders. Translational Psychiatry, 10 (1), 373-373. doi: 10.1038/s41398-020-01054-3

Guo, Y., Gan, X., Zhou, H., Zhou, H., \& Tang, H. (2020). Fingolimod suppressed the chronic unpredictable mild stress-induced depressive-like behaviors via affecting microglial and NLRP3 inflammasome activation. Life Sciences, 263 , 118582. 
Hemmati, F., Dargahi, L., Nasoohi, S., Omidbakhsh, R., Mohamed, Z., Chik, Z., . . Ahmadiani, A. (2013). Neurorestorative effect of FTY720 in a rat model of Alzheimer's disease: Comparison with Memantine. Behavioural Brain Research, 252 , 415-421. doi: https://doi.org/10.1016/j.bbr.2013.06.016

Hla, T., \& Brinkmann, V. (2011). Sphingosine 1-phosphate (S1P). Neurology, 76 (8 Supplement 3), S3. doi: 10.1212/WNL.0b013e31820d5ec1

Iii, R. (2008). Mitogen-Activated Protein Kinases in Synaptic Plasticity and Memory. Learning and Memory: A Comprehensive Reference, 4 (6), 501-523.

Jentsch, J. D., \& Roth, R. H. (1999). The Neuropsychopharmacology of Phencyclidine: From NMDA Receptor Hypofunction to the Dopamine Hypothesis of Schizophrenia.Neuropsychopharmacology Official Publication of the American College of Neuropsychopharmacology, 20 (3), 201-225.

Jentsch, J. D., \& Taylor, J. R. (2001). Impaired Inhibition of Conditioned Responses Produced by Subchronic Administration of Phencyclidine to Rats.Neuropsychopharmacology, 24 (1), 66-74. doi: https://doi.org/10.1016/S0893-133X(00)00174-3

Kesteren, C. V., Gremmels, H., Witte, L. D., Hol, E. M., Gool, A. V., Falkai, P. G., . . Sommer, I. (2017). Immune involvement in the pathogenesis of schizophrenia: a meta-analysis on postmortem brain studies. Translational Psychiatry, 7 (3), e1075.

Kowiański, P., Lietzau, G., Czuba, E., Waśkow, M., Steliga, A., \& Moryś, J. (2018). BDNF: A Key Factor with Multipotent Impact on Brain Signaling and Synaptic Plasticity.Cellular and Molecular Neurobiology, 38 (3), 579-593. doi: 10.1007/s10571-017-0510-4

Liddelow, S. A., Guttenplan, K. A., Clarke, L. E., Bennett, F. C., Bohlen, C. J., Schirmer, L., . . . Peterson, T. C. (2017). Neurotoxic reactive astrocytes are induced by activated microglia. Nature .

Lilley, E., Stanford, S. C., Kendall, D. E., Alexander, S. P. H., Cirino, G., Docherty, J. R., . . . Ahluwalia, A. (2020). ARRIVE 2.0 and the British Journal of Pharmacology: Updated guidance for 2020. British Journal of Pharmacology, 177 (16), 3611-3616. doi: https://doi.org/10.1111/bph.15178

Lima Giacobbo, B., Doorduin, J., Klein, H. C., Dierckx, R. A. J. O., Bromberg, E., \& de Vries, E. F. J. (2019). Brain-Derived Neurotrophic Factor in Brain Disorders: Focus on Neuroinflammation. Molecular neurobiology, 56 (5), 3295-3312. doi: 10.1007/s12035-018-1283-6

Malberg, J. E., Eisch, A. J., Nestler, E. J., \& Duman, R. S. (2000). Chronic Antidepressant Treatment Increases Neurogenesis in Adult Rat Hippocampus. The Journal of Neuroscience, 20 (24), 9104. doi: 10.1523/JNEUROSCI.20-24-09104.2000

Meier, U.-C., Ramagopalan, S. V., Goldacre, M. J., \& Goldacre, R. (2020). Risk of Schizophrenia and Bipolar Disorder in Patients With Multiple Sclerosis: Record-Linkage Studies. Frontiers in Psychiatry, 11 (662). doi: 10.3389/fpsyt.2020.00662

Miguez, A., García-Díaz Barriga, G., Brito, V., Straccia, M., Giralt, A., Ginés, S., . . Alberch, J. (2015). Fingolimod (FTY720) enhances hippocampal synaptic plasticity and memory in Huntington's disease by preventing p75NTR up-regulation and astrocyte-mediated inflammation. Human Molecular Genetics, 24 (17), 4958-4970. doi: $10.1093 / \mathrm{hmg} / \mathrm{ddv} 218$

Momtazmanesh, S., Zare-Shahabadi, A., \& Rezaei, N. (2019). Cytokine Alterations in Schizophrenia: An Updated Review. Frontiers in Psychiatry, 10 (892). doi: 10.3389/fpsyt.2019.00892

Morris, R. (1984). Developments of a water-maze procedure for studying spatial learning in the rat.Journal of Neuroscience Methods, 11 (1), 47-60. doi: https://doi.org/10.1016/0165-0270(84)90007-4

Nassireslami, E., Nikbin, P., Payandemehr, B., Amini, E., Mohammadi, M., Vakilzadeh, G., . . . Sharifzadeh, M. (2013). A cAMP analog reverses contextual and tone memory deficits induced by a PKA 
inhibitor in Pavlovian fear conditioning. Pharmacology Biochemistry and Behavior, 105 , 177-182. doi: https://doi.org/10.1016/j.pbb.2013.02.016

Norbert, \& Müller. (2018). Inflammation in Schizophrenia: Pathogenetic Aspects and Therapeutic Considerations. Schizophrenia Bulletin .

Pape, K., Tamouza, R., Leboyer, M., \& Zipp, F. (2019). Immunoneuropsychiatry - novel perspectives on brain disorders. Nature Reviews Neurology, 15 (6), 317-328. doi: 10.1038/s41582-019-0174-4

Pollak, T. A., Drndarski, S., Stone, J. M., David, A. S., \& Abbott, N. J. (2018). The blood-brain barrier in psychosis. Lancet Psychiatry, 5 (1), 79-92.

Rahimian, R., Wakid, M., O'Leary, L. A., \& Mechawar, N. (2021). The emerging tale of microglia in psychiatric disorders. Neuroscience \& Biobehavioral Reviews, 131 , 1-29. doi: https://doi.org/10.1016/j.neubiorev.2021.09.023

Sanchez-Alavez, M., Nguyen, W., Mori, S., Wills, D. N., Otero, D., Ehlers, C. L., \& Conti, B. (2019). Time course of microglia activation and brain and blood cytokine/chemokine levels following chronic ethanol exposure and protracted withdrawal in rats. Alcohol, 76 , 37-45. doi: https://doi.org/10.1016/j.alcohol.2018.07.005

Santambrogio, L., Belyanskaya, S. L., Fischer, F. R., Cipriani, B., \& Riese, R. (2001). Developmental plasticity of CNS microglia. Proceedings of the National Academy of Sciences, 98 (11), 6295-6300.

Sekar, A., Bialas, A. R., Rivera, H. D., Davis, A., \& O'Donovan, M. C. (2016). Schizophrenia risk from complex variation of complement component 4. Nature, 530 (7589), 177-183.

Sert, N., Hurst, V., Ahluwalia, A., Alam, S., \& Wuerbel, H. (2020). The ARRIVE guidelines 2.0: updated guidelines for reporting animal research. BMJ Open Science, 4 (1), e100115.

Soulet, D., \& Rivest, S. (2008). Microglia. Current Biology, 18 (12), R506-R508.

Sun, Y., Feng, H., Lei, Z., \& Feng, L. (2016). The sphingosine-1-phosphate analogue, FTY-720, promotes the proliferation of embryonic neural stem cells, enhances hippocampal neurogenesis and learning and memory abilities in adult mice. Br J Pharmacol, 173 (18), 2793-2807.

Sun, Y., Hong, F., Zhang, L., \& Feng, L. (2016). The sphingosine-1-phosphate analogue, FTY-720, promotes the proliferation of embryonic neural stem cells, enhances hippocampal neurogenesis and learning and memory abilities in adult mice.British Journal of Pharmacology, 173 (18), 2793-2807. doi: https://doi.org/10.1111/bph.13557

Upthegrove, R., Manzanares-Teson, N., \& Barnes, N. M. (2014). Cytokine function in medication-naive first episode psychosis: a systematic review and meta-analysis.Schizophrenia Research, 155 (1-3), 101-108.

Valeria, Mondelli, Annamaria, Cattaneo, Martino, Belvederi, . . . Forti. (2011). Stress and Inflammation Reduce Brain-Derived Neurotrophic Factor Expression in First-Episode Psychosis. Journal of Clinical Psychiatry .

Vallieres, L., Campbell, I. L., Gage, F. H., \& Sawchenko, P. E. (2002). Reduced Hippocampal Neurogenesis in Adult Transgenic Mice with Chronic Astrocytic Production of Interleukin-6. Journal of Neuroscience, 22 (2), 486-492.

Woo, J. J., Pouget, J. G., Zai, C. C., \& Kennedy, J. L. (2020). The complement system in schizophrenia: where are we now and what's next? Molecular Psychiatry, 25 (1), 114-130. doi: 10.1038/s41380-019-0479-0

Wu, M. (2013). Inhibition of Adult Hippocampal Neurogenesis by Sustained Interleukin-1beta.Dissertations 8 Theses - Gradworks .

Wu, S.-Y., Pan, B.-S., Tsai, S.-F., Chiang, Y.-T., Huang, B.-M., Mo, F.-E., \& Kuo, Y.-M. (2020). BDNF reverses aging-related microglial activation. Journal of neuroinflammation, 17 (1), 210-210. doi: 10.1186/s12974-020-01887-1 
Zhu, Z., Fu, Y., Tian, D., Sun, N., Han, W., Chang, G., . . . Shi, F.-D. (2015). Combination of the Immune Modulator Fingolimod With Alteplase in Acute Ischemic Stroke.Circulation, 132 (12), 1104-1112. doi: doi:10.1161/CIRCULATIONAHA.115.016371

Figure 1

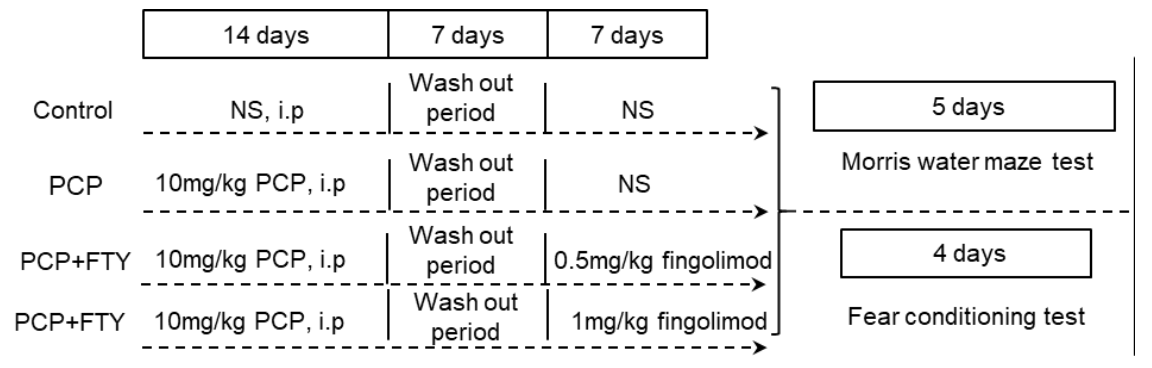

FIGURE 1. Timeline of the experimental procedure. NS, normal saline; FTY, fingolimod; PCP, phencyclidine.

Figure 2

A
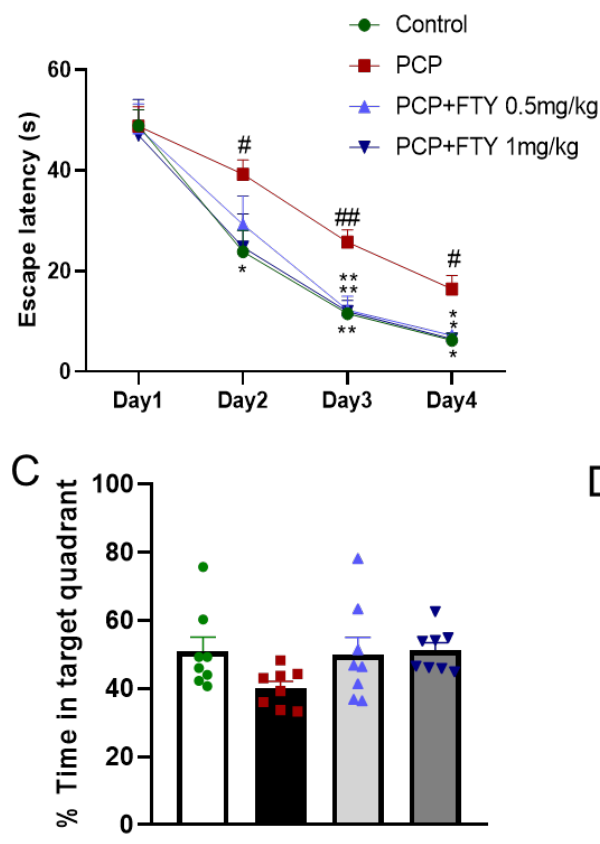

B

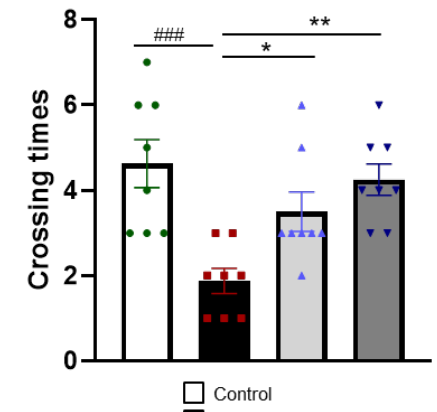

$\mathrm{D}$

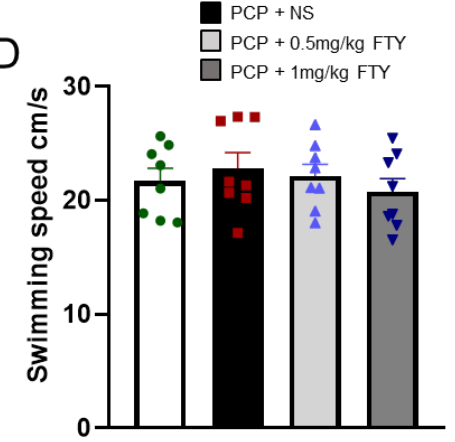

FIGURE 2 Fingolimod attenuated PCP-induced spatial memory deficit in rats in the Morris water maze. (A) The escape latency to find the platform during the training. (B) Crossing times of the virtual platform during the probe test. (C) The percentage of time in target quadrants in the probe test. (D) Swimming speeds of rats in the probe test. Data were shown as means \pm SEM, $\mathrm{n}=8$ per group; \#\#\# 
$\mathrm{P}<.001$ versus Control. ${ }^{*} \mathrm{P}<.05,{ }^{* *} \mathrm{P}<.01$, versus PCP. FTY, fingolimod; PCP, phencyclidine; SEM, standard error of the mean.

Figure 3

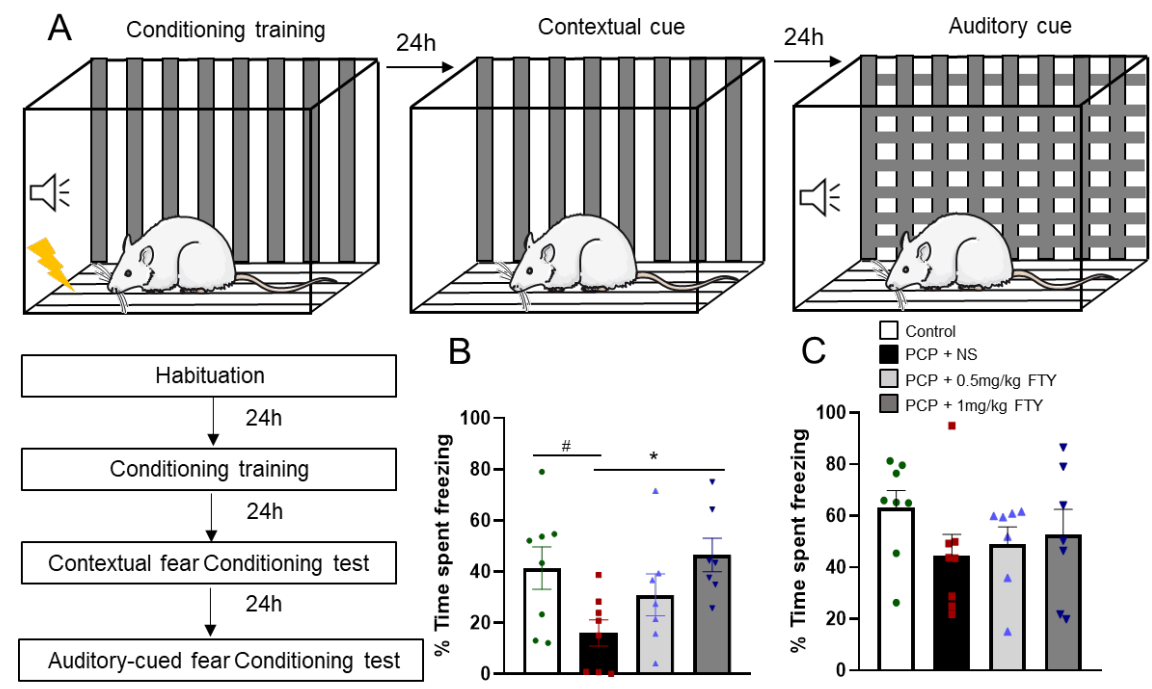

FIGURE 3 Fingolimod attenuated PCP-induced contextual and tone memory retention deficits in rats in the fear conditioning task. (A) Experimental design of the fear conditioning paradigm. (B) Total percentage of freezing in the contextual fear conditioning test. (C) Total percentage of freezing by the auditory-cued fear conditioning test. Two rats were excluded as explained in the methods section. (Control, $\mathrm{n}=8 ; \mathrm{PCP}, \mathrm{n}=8 ; \mathrm{PCP}+0.5 \mathrm{mg} / \mathrm{kg} \mathrm{FTY}, \mathrm{n}=7 ; \mathrm{PCP}+1 \mathrm{mg} / \mathrm{kg} \mathrm{FTY}, \mathrm{n}=7)$. Data were shown as means \pm SEM. \# $\mathrm{P}<.05$ versus Control, ${ }^{*} \mathrm{P}<.05$ versus PCP. FTY, fingolimod; PCP, phencyclidine; SEM, standard error of the mean.

Figure 4

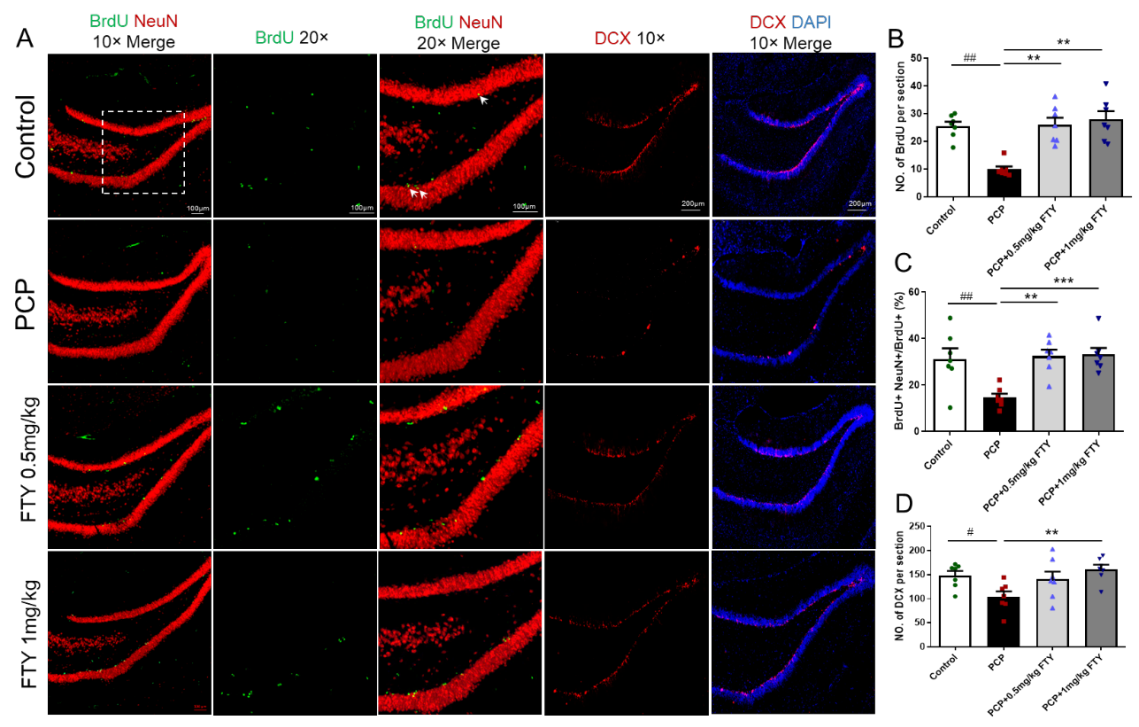


FIGURE 4 Fingolimod treatment enhanced neurogenesis in the dentate gyrus of PCP-treated rats. (A) Confocal microscopic images depict BrdU-labelled newly generated cells (green), NeuN-labelled mature neuron (red), DAPI (blue), and DCX-labelled immature neuron (red) in the dentate gyrus. (B) Quantification of the BrdU-positive cell numbers in the dentate gyrus. (C) Quantitative analysis of the percentage of BrdU/NeuN double-stained cells (as indicated in arrows) in the dentate gyrus. (D) Quantitative analysis of DCX-positive cell numbers in the dentate gyrus. Scale bar $=100 \mu \mathrm{m}$. Data were showed as means $\pm \mathrm{SEM}, \mathrm{n}=7$ per group, $\# \mathrm{P}<.05, \# \# \mathrm{P}<.01$, versus control, ${ }^{* *} \mathrm{P}<.01,{ }^{* * *} \mathrm{P}<.001$ versus $\mathrm{PCP}$. FTY, fingolimod; PCP, phencyclidine; SEM, standard error of the mean.

\section{Figure 5}
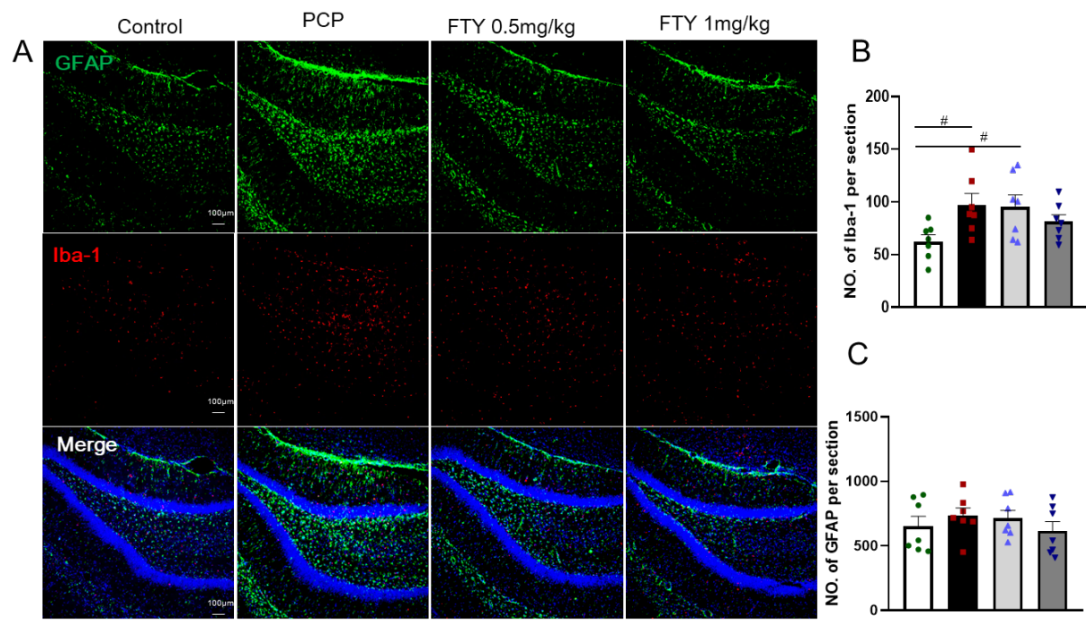

FIGURE 5 Fingolimod treatment inhibited microglial activation in the dentate gyrus of PCPtreated rats. (A) Confocal microscopic images depict Iba-1 labelled microglia (red), GFAP-labelled astrocytes (green), and DAPI (blue) in the dentate gyrus. Scale bar $=100 \mu \mathrm{m}$. Quantitative analysis of Iba-1-positive cells (B) and GFAP-positive cells (C) in the dentate gyrus ( $\mathrm{n}=7$ per group). Data were shown as means $\pm \mathrm{SEM}, \# \mathrm{P}<.05$ versus control. FTY, fingolimod; Iba-1, ionized calcium binding adapter molecule 1; GFAP, glial fibrillary acidic protein; PCP, phencyclidine; SEM, standard error of the mean.

\section{Figure 6}

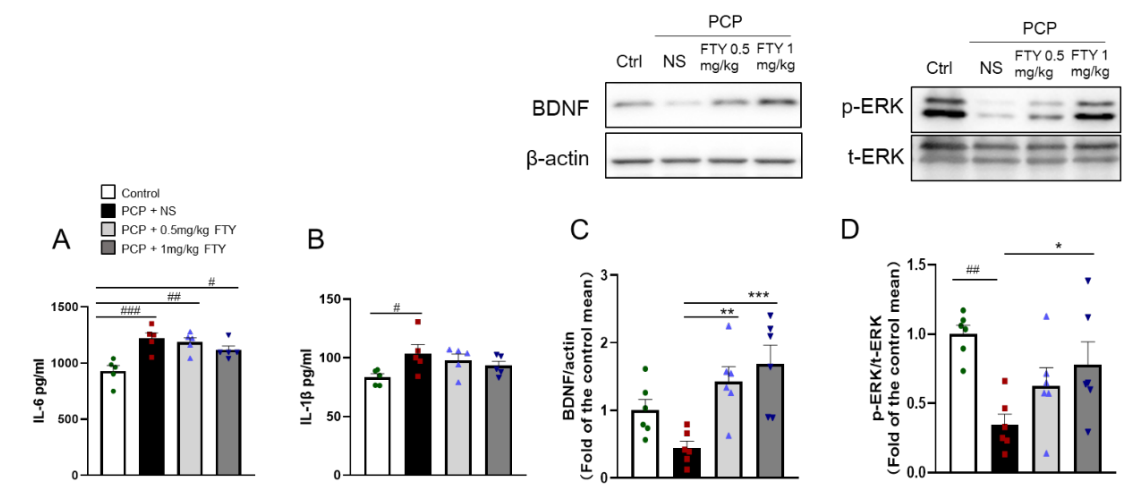

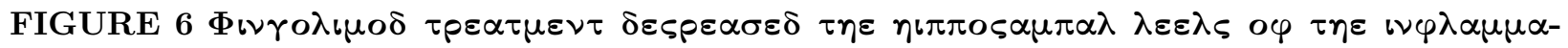




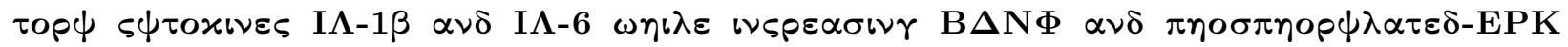
$\varepsilon \xi \pi \rho \varepsilon \sigma \sigma\llcorner\mathrm{\nu}$. Multiplexed flow cytometric assay of IL-6 (A) and IL-1 $\beta$ (B) levels in the hippocampus (n=5 per group). Representative images of western blotting and (C) protein expression of BDNF and $\beta$-actin as internal controls. (D) Expression of phosphorylated-ERK and total ERK in the hippocampus ( $\mathrm{n}=6$ per group). Data are shown as means $\pm \mathrm{SEM}, \# \mathrm{P}<.05$, \#\#P $<.01$ versus control, \#\#\#P $<.001$ versus control, ${ }^{*} \mathrm{P}<.05,{ }^{*} \mathrm{P}<.01,{ }^{* * *} \mathrm{P}<.001$ versus $\mathrm{PCP}$. BDNF, brain-derived neurotrophic factor; ERK, extracellular signal-regulated kinase; p-ERK, phosphorylated-ERK; t-ERK, total ERK; PCP, phencyclidine; SEM, standard error of the mean. 